\title{
Medidas de mitigación para el acuífero costero La Yarada, un sistema sobreexplotado en zonas áridas
}

\author{
Mitigation measures for the La Yarada coastal aquifer, \\ an overexploited system in arid areas
}

Edwin Pino V. ${ }^{*}$, Lia Ramos F. ${ }^{2}$, Jesús Mejía M.², Eduardo Chávarri V. ${ }^{2}$, David Ascensios $T^{2}$

\section{RESUMEN}

Este artículo es una revisión del estado actual de la sobreexplotación del acuífero costero La Yarada, emplazado en una zona árida, cabecera del Desierto de Atacama. Según referencia de otros sistemas similares a nivel mundial, se ha identificado las medidas de mitigación más favorables para la conservación del sistema acuífero. Se plantean acciones físicas y no físicas o de gestión, conducentes a la mitigación del colapso y lograr la recuperación y conservación del acuífero.

Palabras clave: acuífero La Yarada, medidas de mitigación, conservación del acuífero.

\begin{abstract}
This article is a review of the current status of the overexploitation of the La Yarada coastal aquifer, located in an arid area, head of the Atacama Desert. According to references from other similar systems worldwide, the most favorable mitigation measures for the conservation of the aquifer system have been identified. Physical and non-physical or management actions are proposed, leading to the mitigation of collapse and achieving the recovery and conservation of the aquifer.
\end{abstract}

Key words: La Yarada aquifer, mitigation measures, aquifer conservation.

\section{Introducción y revisión bibliográfica}

Casi la mitad de la población mundial se abastece de agua potable por medio de fuentes subterráneas y se estima que el $20 \%$ de los acuíferos está siendo sobreexplotado (WWAP, 2015). Un estimado de ocho a diez millones de kilómetros cúbicos $\left(\mathrm{km}^{3}\right)$ de agua subterránea fresca representan la mayor parte del total de agua dulce líquida en la Tierra, o para ser más precisos: aproximadamente del 98 al 99\% de ella (Shiklomanov y Rodda, 2004); en comparación, el volumen global de agua dulce en los lagos es inferior al $1 \%$ del volumen total de agua dulce subterránea (Shiklomanov y Rodda, 2004; Schneider et al., 2011; Singh, 2014). Existe una gran cantidad de evidencia científica que documenta el uso excesivo de las aguas subterráneas en todo el mundo. A pesar de esta evidencia, muchos y quizás la mayoría de los acuíferos no se manejan de manera sostenible (Garner, 2019).

La sobreexplotación del agua subterránea y la sobreexplotación de acuíferos son conceptos que se están convirtiendo en términos de uso común en gestión hídrica (Custodio, 2002). Muchos hidrólogos, gestores y periodistas los usan para referirse a un acuífero explotado intensamente o que presenta situaciones conflictivas. La sobreexplotación se puede definir como la situación en la que durante varios años la extracción media de agua subterránea de un acuífero supera o se aproxima a la recarga media.

En la actualidad se han dedicado numerosos estudios a la optimización de estrategias de gestión de aguas subterráneas en acuíferos costeros. Sin embargo, poco se ha hecho para abordar el problema de los acuíferos contaminados (Abarca et al., 2006). La difusión de las herramientas de

\footnotetext{
1 Universidad Nacional Jorge Basadre Grohmann, Tacna, Perú.

2 Universidad Nacional Agraria La Molina, Lima, Perú.

* Autor por correspondencia: epino68@hotmail.com, epinov@unjbg.edu.pe
} 
tecnología de la información y el aumento de la producción de datos en la gestión de las aguas subterráneas permitió el desarrollo de diferentes sistemas de información (Laraichi et al., 2016; Sanchez y Eckstein, 2017).

En circunstancias de cambio climático, el agua subterránea se vuelve particularmente importante; de hecho, cuanto más se amenaza el agua superficial a través de las sequías y la salinización; más necesario se hace comprender y administrar mejor los recursos de agua subterránea (Margat y Gun, 2013). Los cambios en la disponibilidad hídrica de los desiertos debido a los humanos tienen implicancias importantes desde un nivel local, regional o global (Mahowald, 2007). Las regiones áridas tienen un clima y un entorno ecológico frágil y sensible. Por ello, los recursos hídricos son extremadamente necesarios para mantener un equilibrio ecológico (Zhang, 2000; Wang y Qin, 2017).

Diferentes tipos de aprovechamientos de los acuíferos como el hidrogeológico termal, municipal, industrial o agrícola, lo transforman, por un lado, en un riesgo potencial de sobreexplotación de un recurso natural, y así, simultáneamente, en un factor de riesgo para el desencadenamiento de complejos conflictos sociales (Babaye et al., 2018). En el acuífero costero La Yarada, la crisis de gobernabilidad y gobernanza en el uso del agua subterránea es un elemento que contribuye al agotamiento y deterioro de su calidad, por procesos de intrusión marina en sus más de 100 años de explotación (Pino et al., 2017; Pino et al., 2018; Pino, 2019; Pino et al., 2019). Más aún, se ha provocado que la calidad del agua pase de aceptable a mala, evidenciando un deterioro que está relacionado con el incremento de los volúmenes de explotación y la disminución de la recarga secundaria de agua de buena calidad (Pino y Coarita, 2018). A su vez, una fuerte explotación de las aguas subterráneas de los acuíferos costeros, provoca el secado de manantiales (Vernoux et al., 2018).

En este trabajo, buscamos establecer la situación actual de sobreexplotación del acuífero costero La Yarada como sustento de una agricultura en zonas áridas y determinar cuáles serían las medidas de mitigación para evitar su colapso.

\section{Metodología}

Según las características de este caso, establecimos una revisión de documentos existentes en casi todo el historial de explotación del acuífero costero La Yarada, ubicado en la región Tacna al sur del Perú (Figura 1), se consultaron estudios académicos y material oficial de dominio público de
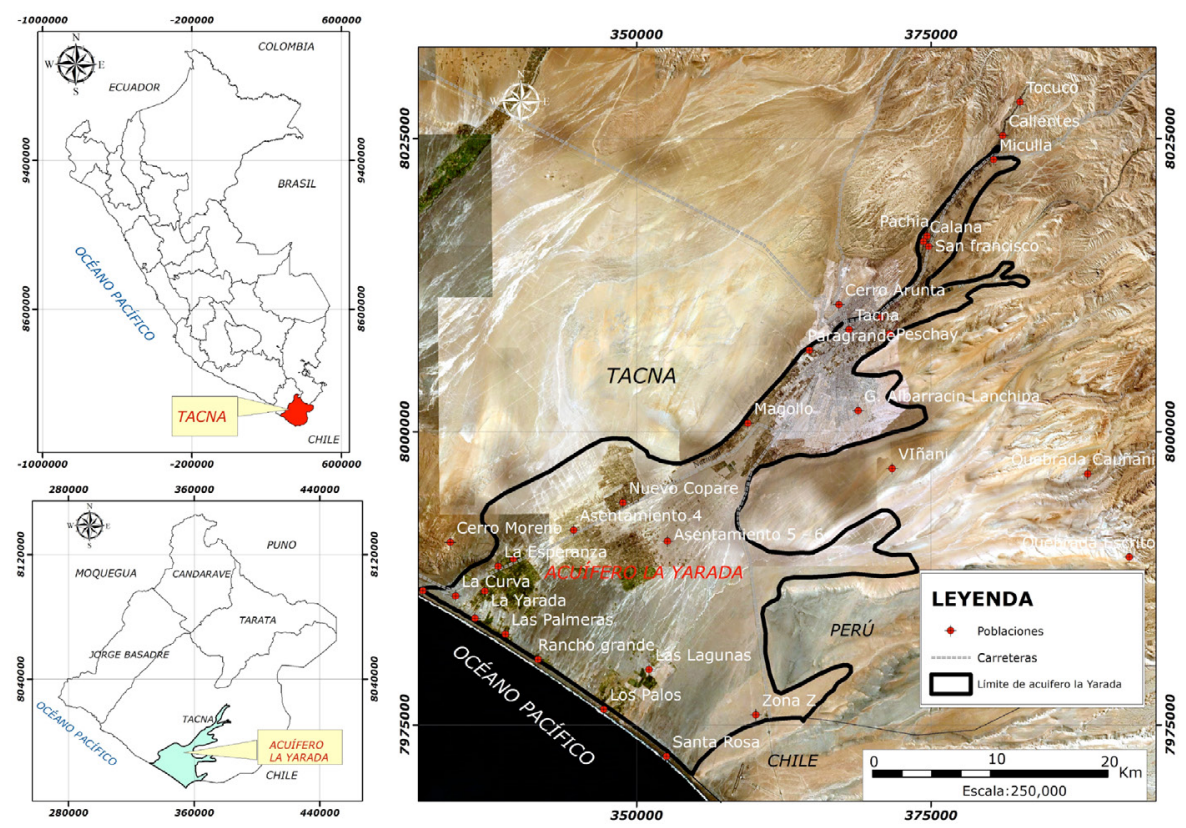

Figura 1. Mapa de ubicación del acuífero La Yarada. 
instituciones académicas, tecnológicas y científicas. Se busca mediante este análisis, establecer las mejores medidas ingenieriles y de gestión. Esta información recopilada y analizada sirvió de base para establecer la discusión y conclusiones sobre las medidas de mitigación a proponer en este sistema acuífero sobreexplotado.

\section{Situación actual del sistema acuífero}

El acuífero La Yarada, se encuentra emplazado en la región Tacna, límite con Chile, y a su vez cabecera del desierto de Atacama. Corresponde a una región con déficit hídrico, las descargas medias de los ríos en la vertiente del Pacífico en la región son muy bajas respecto a los ríos de la costa central y norte del país tal como se puede apreciar en la Figura 2.

Esta situación de déficit se ve acrecentada puesto que este acuífero no tiene un programa de operación, las extracciones de agua se dan al margen de la normatividad vigente, no existiendo ningún tipo de control y no es posible identificar a los infractores. Consecuentemente, se está generando un descenso altamente significativo del nivel freático lo que ha ocasionado contaminación del agua dulce por procesos de intrusión marina. Según Pino (2019), se ha identificado el período en el que la explotación ha sido en régimen equilibrado (recarga - extracciones) hasta la década de los 70 y no equilibrado a partir de los 80 .

En la Figura 3 se muestra la superficie bajo riego en el tiempo, tomándose como base imágenes LANSAT 1-5 MSS. Los años corresponden a 1972 con 2040 ha, 1987 con 4407 ha, 2008 con 13500 ha y 2019 con 40511 ha. La evolución histórica de la superficie regada y los volúmenes explotados en el acuífero, se muestra en la Figura 4 y en la Figura 5 se aprecia el balance del sistema acuífero en función del tiempo, evidenciando un período inicial de equilibrio y posteriormente un desbalance que cada vez se hace más negativo.

Los balances hídricos reportados corresponden a diferentes instituciones, en 1965 el Instituto Nacional de Investigación y Fomento Minero (INIFM) observó que el acuífero La Yarada arroja un superávit anual de $36,0 \mathrm{hm}^{3}$. Para el año 1989, el Proyecto Especial Tacna (PET), adscrito al Instituto Nacional de Desarrollo (INADE), registra un balance negativo que asciende a los $-15,5 \mathrm{hm}^{3}$. Asimismo, en el año 2009, el Instituto Geológico, Minero y Metalúrgico (INGEMMET), concluyen
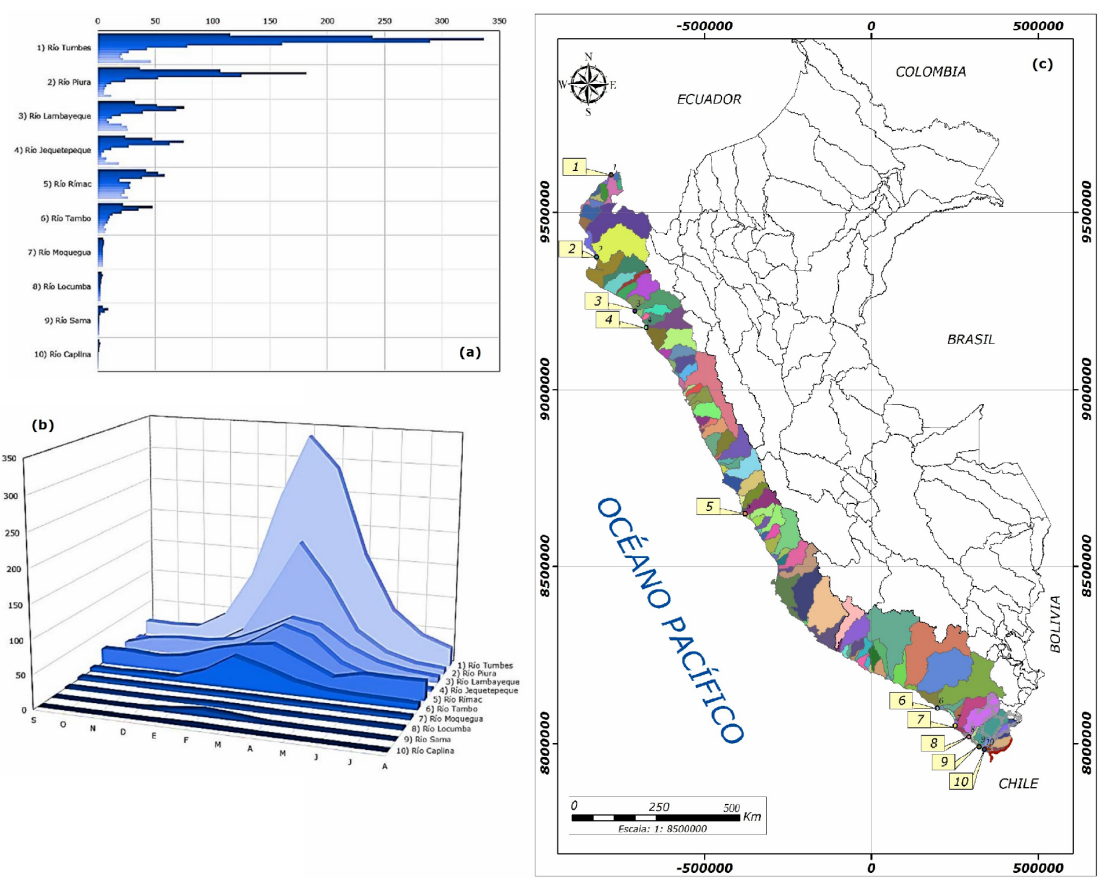

Figura 2. Descargas de los ríos de la costa peruana, vertiente del Pacífico. 

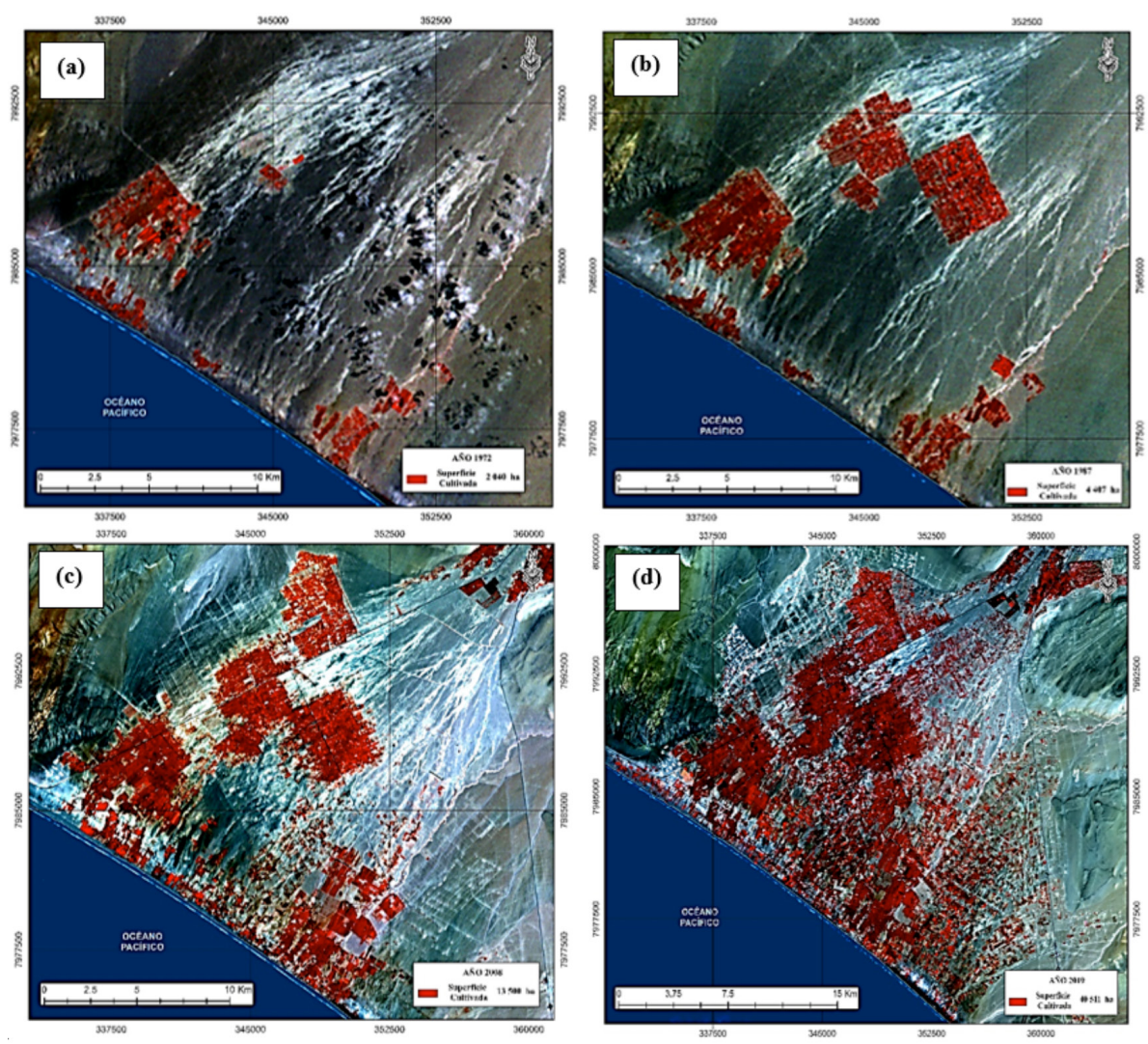

Figura 3. Superficie irrigada en diferentes años, en base a imágenes LANSAT 1-5 MSS. (a) 1972, 2040 ha, (b) 1987, 4407 ha, (c) 2008, 13500 ha, (d) 2019, 40511 ha.
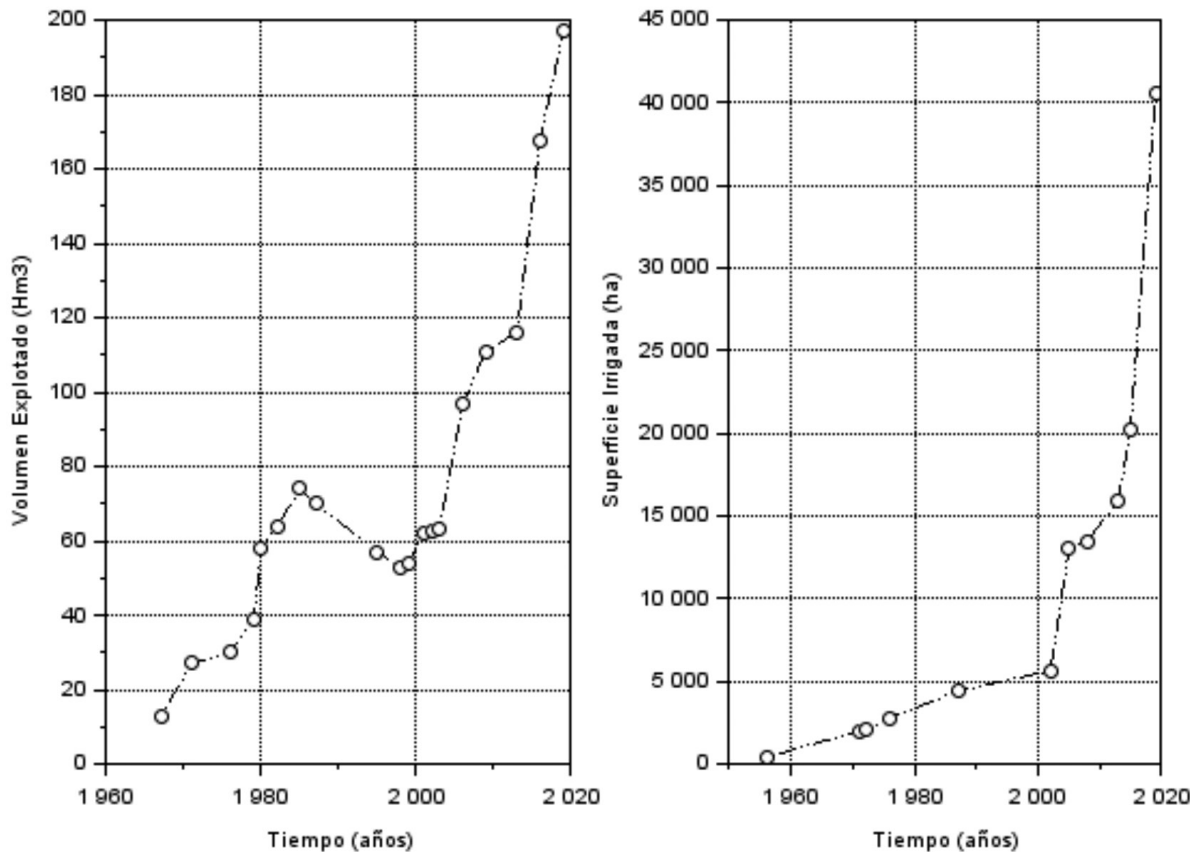

Figura 4. Evolución de la superficie irrigada y los volúmenes explotados al año 2019. 


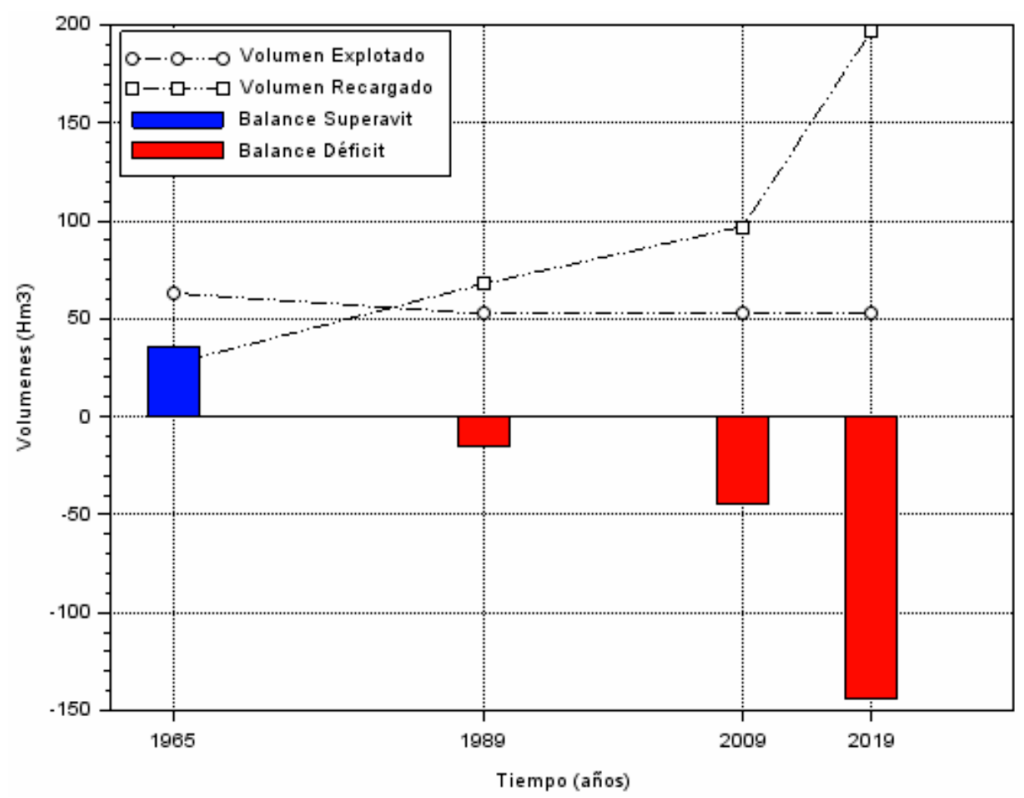

Figura 5. Evolución del balance hídrico en el acuífero.

que el acuífero se encuentra en desbalance con $-44,0 \mathrm{hm}^{3}$ y finalmente el año 2019, la Autoridad Nacional del Agua (ANA) concluyen que el acuífero está en desbalance con $-144,09 \mathrm{hm}^{3}$, cifras que denotan el deterioro progresivo del sistema.

Este acuífero como la mayoría de ellos en el mundo presentan problemas de cantidad, calidad, gobernanza y gobernabilidad. Según Pino et al., (2018), esta crisis en el uso del agua subterránea en el acuífero costero La Yarada, es un elemento que contribuye al agotamiento y al deterioro de la calidad del agua subterránea, por procesos de intrusión marina. Este problema, en todos sus términos, altera y excede las leyes relativas a los regímenes de gestión y las limitaciones en la disponibilidad de recursos hídricos subterráneos, si el aparato gubernamental no toma medidas inmediatas, la crisis del sistema podría volverse irreversible con el consiguiente impacto económico que esto conlleva.

En el acuífero La Yarada, la extracción de agua se da para intensificar la actividad agrícola, esto mantiene colapsado el sistema de aguas subterráneas, un acuífero sobreexplotado y una clara desconexión de las aguas subterráneas con las superficiales, lo que acrecienta más aún el problema.

Otros elementos no ligados directamente a las extracciones de agua, son descritas por Chávez et al. (2012), quienes concluyen que en los acuíferos costeros, el ascenso del nivel del mar, derivado del cambio climático, provocará varios efectos: (i) la pérdida por salinización de la porción de ellos ubicada en el área invadida por el mar; (ii) el avance tierra adentro de la interface salina agua dulce/agua salada, hacia una nueva posición de equilibrio; (iii) la disminución del espesor saturado de agua dulce en la porción ocupada por la interface salina; (iv) la reducción de la disponibilidad y de la reserva almacenada de los acuíferos, y (v) la inutilización o salinización de los pozos ubicados en la faja afectada por el fenómeno.

Identificado el problema, podemos establecer una estrategia para frenar el proceso de contaminación del acuífero y dictar medidas que nos permitan recuperar el nivel freático del mismo, así como su calidad. Según las medidas que se vienen utilizando en el mundo, podemos establecer un conjunto de medidas de orden estructural y no estructural, dentro de las medidas estructurales tenemos un grupo convencionales, no convencionales y especiales o alternativas. En el primer caso tratándose de medidas convencionales podemos citar algunos elementos como el empleo de embalses, explotación de aguas subterráneas y transferencia de agua entre cuencas. En el segundo grupo de medidas no convencionales; podemos establecer aquellas que están orientadas a establecer reúso y reciclaje de agua y desalinización. Mientras 
que el tercer grupo de medidas especiales o alternativas; nos referimos a la administración de la recarga de los acuíferos y técnicas paliativas como son: disminución de escorrentía en bosque y zonas urbanas, trampas para escorrentía, ahorro, redes de tuberías eficientes, disminución de evaporación en los embalses, etc.

\section{Medidas de mitigación no estructurales}

Es necesario integrar los datos disponibles de todas las fuentes para obtener información relevante en diferentes niveles del proceso de toma de decisiones, muchas investigaciones intentan abordar este problema, la integración de datos para la construcción de un almacén de datos espaciotemporal es fundamental para apoyar en la toma de decisiones en la gestión del agua subterránea (Laraichi et al., 2016). Diferentes instituciones públicas y privadas vienen desarrollando a lo largo del tiempo estudios e investigaciones sobre el comportamiento del acuífero, en tal sentido urge la necesidad, en este caso podría ser la Autoridad Nacional del Agua (ANA) quien integre toda la información disponible, sistematice y la ponga a disposición de los usuarios e instituciones que pretendan proponer medidas correctoras de uso racional del agua subterránea en este acuífero.

En este sentido, es importante establecer un Plan de Gestión y Reordenamiento de Extracciones, en el acuífero La Yarada, no hay ningún control sobre las extracciones, por tanto, urge la necesidad de elaborar un plan de esta naturaleza, partiendo del saneamiento físico-legal de la tierra, lo cual es un proceso de formalización de uso que tiene que llevarse a cabo tomando como base información de fotografías aéreas de "drone" e imágenes satelitales a una fecha de cierre preestablecida.

\section{Medidas de Mitigación Estructurales}

La cuenca Caplina donde se encuentra emplazado el sistema acuífero, es deficitaria en recursos hídricos, actualmente viene presentando problemas de descensos de niveles freáticos e intrusión marina con el consiguiente proceso de salinización del agua subterránea. En tal sentido, se hace necesario plantear un conjunto de alternativas ingenieriles que nos permita recuperar el nivel freático del acuífero, y por ende la calidad de su agua. Estas medidas corresponden a trasvases, tratamiento de laderas, reutilización de aguas residuales y la implementación de barreras hidráulicas (Figura 6).

\section{Trasvase entre cuencas}

Se dispone de proyectos existentes, cuya finalidad, es dar solución a la escasez de recursos hídricos de la región Tacna, la optimización de dichos recursos y de mejorar el manejo del agua en las cuencas, gestionando su uso con eficiencia y racionalidad, mediante la ejecución de proyectos de trasvase (cuenca alto andina Maure a las cuencas de la vertiente del Pacífico; Sama, Locumba y Caplina). En consecuencia, sus componentes principales corresponden a trasvase agua de las cuencas de la vertiente del Lago Titicaca a la vertiente del Pacífico. La propuesta de trasvase trata sobre la incorporación del agua que se lograra con los proyectos Vilavilani y Yarascay como fuentes para la inyección al sistema acuífero (Figura 7).

\section{Tratamiento de laderas}

Según Vásquez (2011), utilizadas para inducir infiltración no solo beneficia la recarga de los acuíferos sino que también contribuyen a mitigar los efectos de los "huaycos" aguas abajo. Sabemos que, en las laderas, ubicadas sobre los $2000 \mathrm{msnm}$, se dan precipitaciones medias entre 400 y 1400 mm/año (entre diciembre y marzo), estas precipitaciones generan volúmenes hasta de $14000 \mathrm{~m}^{3} /$ ha, de lo cual entre el 60 y el $80 \%$ escurre hacia las quebradas y ríos. Asimismo, parte de esta agua puede ser inducida a infiltrarse llegando más adelante a formar parte del agua subterránea. La entrada de agua desde la zona no saturada a la zona saturada se define como la recarga de agua subterránea. Esta ocurre solo cuando el agua fluye debajo del suelo y se infiltra en la zona saturada (Singh et al., 2019). Varios factores afectan la ocurrencia y el movimiento del agua subterránea, como litología, estructura geológica, pendiente, patrón de drenaje, forma del terreno, uso del suelo, cobertura del suelo y clima.

Se puede recurrir a la construcción de pequeños o medianos reservorios o embalses en la zona media y alta de las cuencas donde existen condiciones topográficas naturales adecuadas para convertirlas o utilizarlas como pequeños o medianos embalses o reservorios de agua; con bajas inversiones, pero con gran efecto e impacto social, económico y 


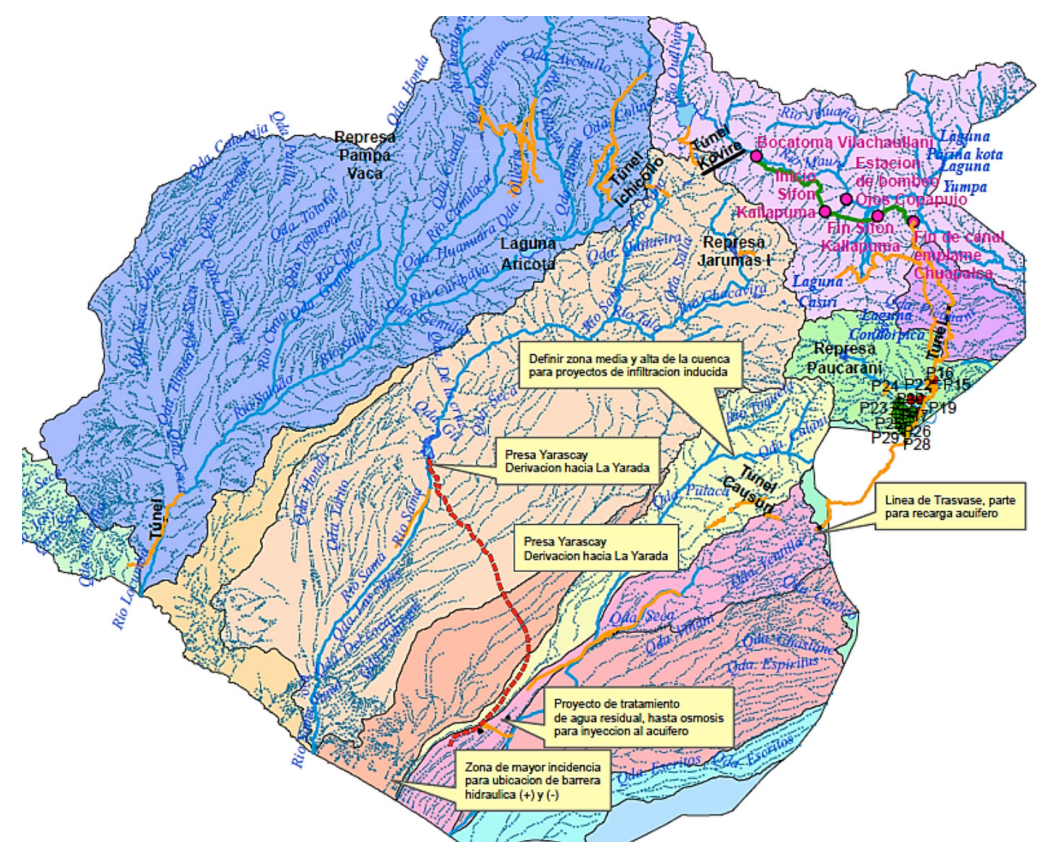

Figura 6. Esquema de ubicación de medidas estructurales.

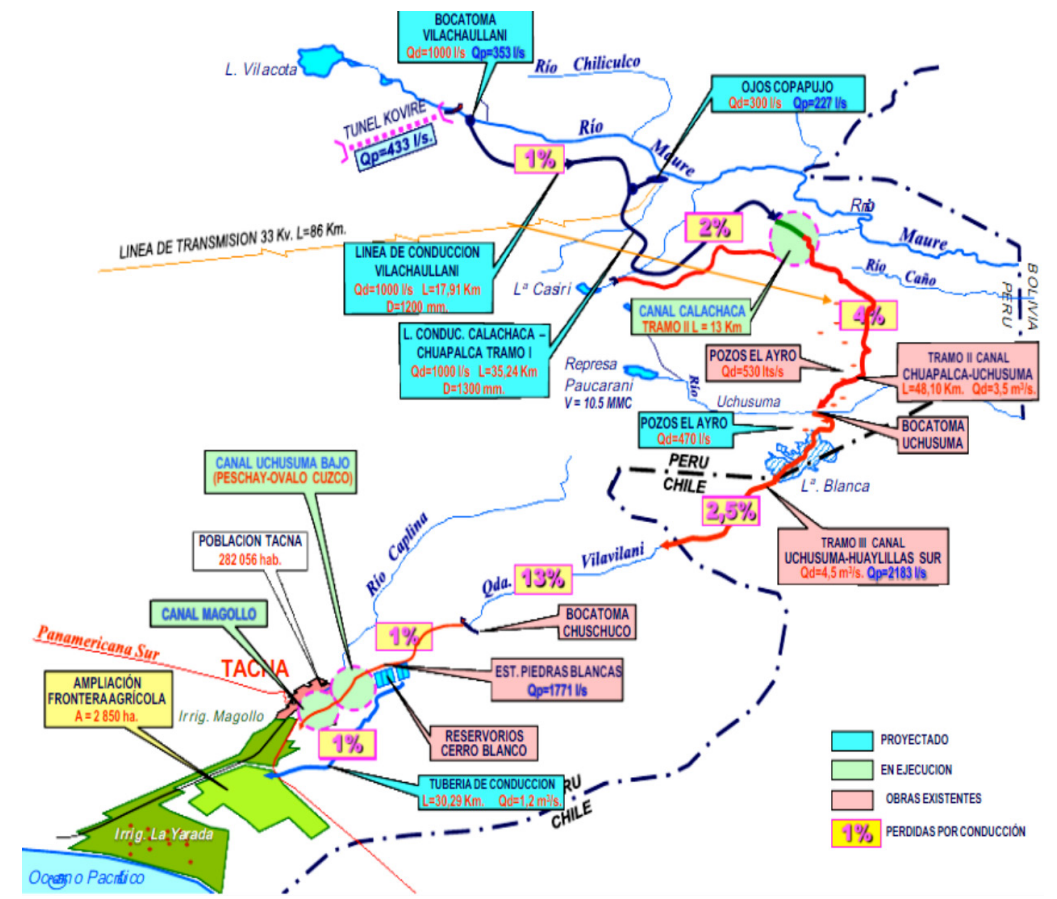

Figura 7. Esquema hidráulico del Proyecto Vilavilani (Fuente: Proyecto Especial Tacna).

ambiental. También es posible el incremento de la capacidad de almacenamiento de lagunas naturales existentes. Asimismo, la construcción de zanjas de infiltración en el sentido trasversal a la pendiente del terreno puede hacerse en forma manual o mecanizada, dependiendo de las condiciones del terreno, estas zanjas inducen la infiltración de gran cantidad de agua que proviene de las lluvias. 
La reforestación es una actividad sumamente eficiente en el proceso de control de la erosión y el incremento de la infiltración del agua de lluvia. Esta actividad es mucho más eficiente si se complementa con las zanjas de infiltración para captar el agua de escurrimiento superficial y facilitar su infiltración. De la misma manera la regeneración o plantación de pastizales, es una tarea fundamental que debe llevarse a cabo para inducir la infiltración del agua de lluvia, traerá consigo la reducción de la erosión debido a la reducción en la escorrentía superficial.

A su vez, el aprovechamiento de las fallas geológicas es una práctica ancestral utilizada desde la época de los incas y conocida como "amunas", consiste en ubicar zonas con fallas geológicas o verdaderos "traguaderos de agua" en las partes altas y medias de las cuencas. Se capta el agua de riachuelos o corrientes efímeras para ser conducidas por canales hasta la falla geológica y de esta manera inducir la recarga del sistema subterráneo.

\section{Reutilización de aguas residuales}

Con las crecientes presiones sobre los recursos hídricos, surgen preocupaciones sobre cómo encontrar nuevos recursos capaces de ayudar a alcanzar el equilibrio entre la demanda y la oferta. En este contexto, una de las principales posibilidades para hacer frente a la escasez de agua es la recuperación y reutilización de aguas residuales (Salgot y Folch, 2018), esta reutilización la podemos orientar a la recarga artificial del acuífero, para lo cual es importante establecer los lineamientos de la calidad del agua a ser inyectada al acuífero. El agua procedente de la EPS Tacna deberá ser sometida, después del tratamiento secundario, a tratamiento terciario, consistente en coagulación-floculación lastrada, decantación lamelar, filtración y desinfección por UV y cloro. En esta planta se deben realizar tratamientos adicionales de ultrafiltración, ósmosis inversa (sólo al 50\% del agua) y desinfección por UV, produciéndose el caudal que se distribuiría a los pozos de inyección (Figura 8).

\section{Implementación de barreras hidráulicas}

La recuperación y reutilización del agua se han convertido en componentes esenciales de la gestión de los recursos hídricos y los mecanismos para su incorporación en el sistema acuífero (Post, 2005). Pool y Carrera, (2010), proponen una terminología para clasificar las barreras hidráulicas en cuatro tipos: baja permeabilidad subsuperficial, positiva, negativa y mixta (Figura 9). Las barreras subterráneas de baja permeabilidad (Figura 9a) consisten en paredes verticales (paredes de lodo, tablestacas de acero u hormigón) colocada tierra adentro para bloquear la intrusión de agua de mar. Este sistema requiere una ingeniería considerable e inversión. Además, puede ser contraproducente si se detiene el bombeo o existen fuentes de contaminación. Las barreras hidráulicas positivas (Figura 9b) inyectan agua en el acuífero, que levanta la carga piezométrica, por lo tanto, evitan que el agua salada fluya tierra adentro. Las barreras hidráulicas negativas (Figura 9c) bombean cerca de la orilla, interceptando así la entrada de agua salada. La intrusión de agua de mar está impedida siempre que las bombas de la barrera estén operando. Las barreras mixtas (Figura 9d) son sistemas de bombeo por inyección, este sistema puede usarse para inyectar agua dulce en profundidades bajas mientras bombea agua salada a profundidad, válido para acuíferos de gran espesor y/o verticalmente anisotrópicos (Thangarajan, 2007).

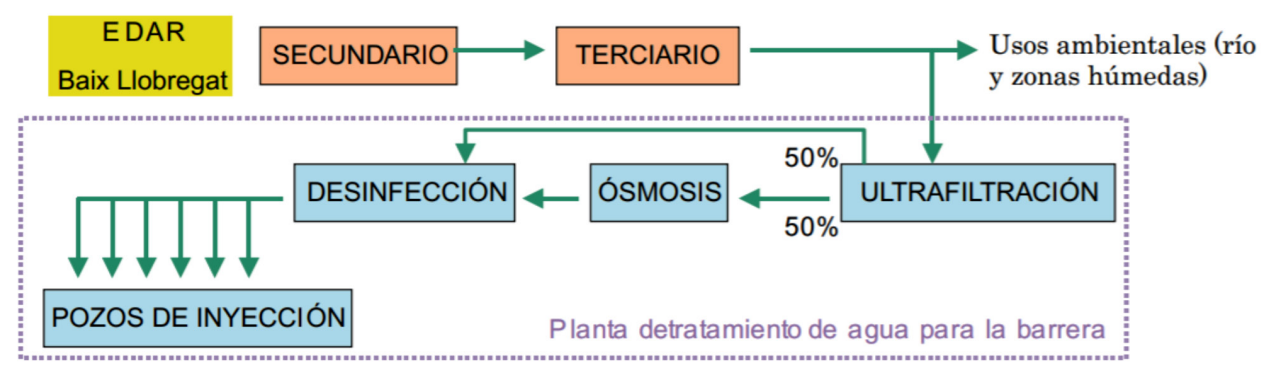

Figura 8. Tratamientos del agua previos a su inyección en el acuífero, efluente terciario de la EDAR del Baix Llobregat, Barcelona, España. 
a) Low permeability subsurface barrier

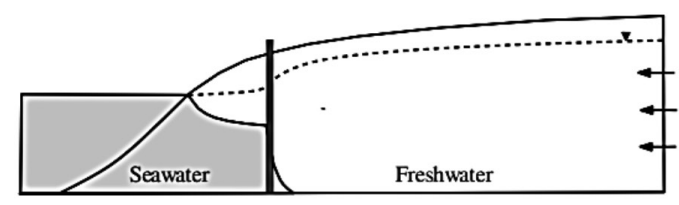

c) Negative hydraulic barrier

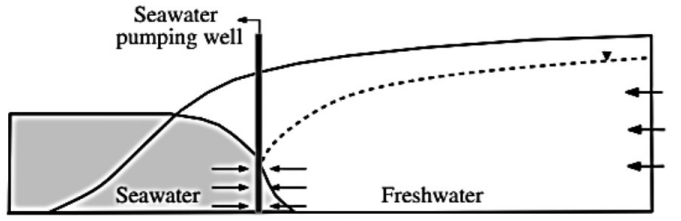

b) Positive hydraulic barrier

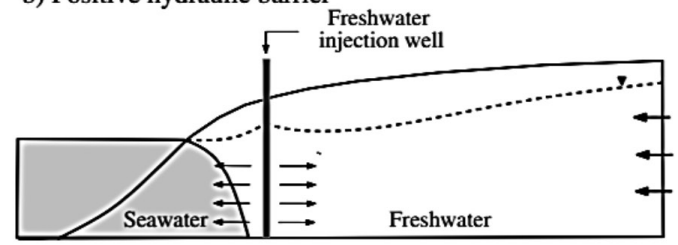

d) Mixed barrier

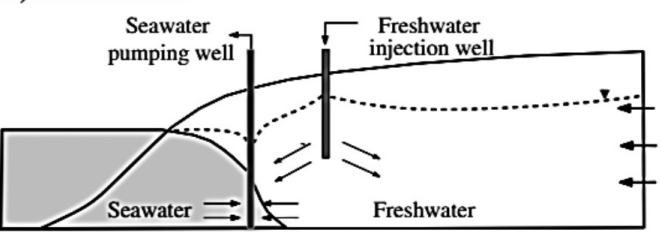

Figura 9. Tipos de barreras hidráulicas (a) baja permeabilidad subsuperficial, (b) positiva, (c) negativa, (d) mixta.

El acuífero La Yarada, presenta condiciones favorables para establecer barreras hidráulicas de cualquiera de los tipos conocidos, a la margen derecha e izquierda del cono de deyección se dispone de una barrera impermeable rocosa, que garantizaría su funcionamiento.

\section{Discusión y aportes}

Durante los más de 100 años de explotación, del acuífero La Yarada, se conoce que, hasta la década de los años 70, estaba equilibrada entre recarga y extracciones. A partir de la década de los 80 , se sabe que la sobreexplotación del acuífero ha provocado descensos del nivel freático y que la potabilidad del agua considerada aceptable cambie a mediocre, evidenciando el deterioro en la calidad del agua (Pino, 2019).

Según la disponibilidad hídrica en la zona de estudio, debemos tomar en consideración todas las medidas citadas, tanto las estructurales como las no estructurales (de gestión o reordenamiento y las ingenieriles). En cuanto a las medidas no estructurales, su implementación es un paso primordial e importante para iniciar la recuperación del acuífero La Yarada. Se requiere reordenar el uso del agua subterránea y establecer los lineamientos de formalización de uso de agua, acorde a la legislación vigente.

Se proponen medidas de remediación basadas en la recarga artificial del acuífero con la utilización de: trasvases, tratamiento de laderas, reutilización de aguas servidas, desalación, así como avanzar en el camino para iniciar nuevas líneas de investigación que desarrollen la técnica de recarga artificial de acuíferos. Asimismo proponemos incorporar criterios de educación ambiental, sensibilización y divulgación, aplicados a la conservación del acuífero.

Dentro de las alternativas planteadas hay algunas que son de aplicación rápida y directa, pero de sostenibilidad compleja, como es el caso de las barreras hidráulicas, que requieren costos altos de operación, situación que hace compleja su aplicabilidad. En cuando al uso de aguas residuales, es una alternativa que consideramos importante, para lo cual se debe fijar la reserva de cuotas permanentes de agua residual con fines de tratamiento e inyección al acuífero.

Respecto al tratamiento de laderas, su aporte de agua es considerable y además trae beneficios colaterales como evitar la pérdida de suelos entre otros. En cuanto al agua de trasvase, es la alternativa que más estudios y avance físico en obras tiene en la actualidad, en Tacna se viene trabajando en estos proyectos hace muchos años y además cuentan con avance físico de obras, lo que posibilita que esta alternativa sea importante en el proceso de recuperación del acuífero, pensando en la inyección de agua al sistema o simplemente el uso en superficie con la reducción de extracciones que requiere el sistema para mejorar su balance actual.

\section{Conclusiones}

Se expusieron evidencias claras del estado actual de sobreexplotación del acuífero La Yarada, al igual que muchos acuíferos del mundo requiere de medidas urgentes para su recuperación antes de llegar al colapso. En la actualidad el proceso de contaminación por intrusión marina se viene 
acrecentando debido a la sobreexplotación del agua subterránea. En este sentido, se plantean dos tipos de medidas correctoras la primera de orden no estructural y la segunda estructurales. Las primeras orientadas a establecer un plan de gestión y reordenamiento de extracciones, elemento de mucha importancia porque no se puede precisar el volumen de extracción, existe mucha resistencia de los usuarios no formalizados a ser auditados. En cuanto a las medidas estructurales las podemos sub agrupar en convencionales y no convencionales, siendo en el primer caso el empleo de embalses y transferencia de agua entre cuencas. Las medidas no convencionales posibles de implementar corresponden a reúso, reciclaje y desalinización. Mientras que el tercer grupo de medidas especiales o alternativas; la administración de la recarga de los acuíferos y técnicas paliativas como: disminución de escorrentía en bosques y zonas urbanas, trampas para escorrentía, ahorro, redes de tuberías eficientes, disminución de evaporación en los embalses, etc. De estas posibles medidas a implementar, la recarga artificial del acuífero combinada con la implementación de barreras hidráulicas son factibles de incorporar en el sistema acuífero, se dispondrá en el mediano y corto plazo de volúmenes de agua trasvasados que pueden ser utilizados para tal fin. Así mismo proponemos incorporar criterios de educación ambiental, sensibilización y divulgación, aplicados a la conservación del acuífero.

\section{Agradecimientos}

Este trabajo ha sido desarrollado en el marco del proyecto de investigación "Integración de métodos hidrodinámicos, hidroquímicos e isotópicos para precisar el funcionamiento y manejo sostenible del acuífero la Yarada, Tacna, Perú", financiado con fondos de canon, sobrecanon y regalías mineras de la Universidad Nacional Jorge Basadre Grohmann, Tacna, Perú.

\section{Literatura Citada}

Abarca, E.; Vázquez-Suñé, E.; Carrera, J.; Capino, B.; Gámez, D.; Batlle, F.

2006. Optimal design of measures to correct seawater intrusion. Water Resources Research, 42 (9). DOI: 10.1029/ 2005WR004524.

Babaye, M.S.A.; Orban, P.; Ousmane, B.; Favreau, G.; Brouyère,

S.; Dassargues, A.

2018. Characterization of recharge mechanisms in a Precambrian basement aquifer in semi-arid south-west Niger. Hydrogeol J., 1-17. DOI: 10.1007/s10040-018-1799-x. Custodio, E.

2002. Aquifer overexploitation: what does it mean? Hydrogeology Journal, 10: 254-277.

Garner, E.L.

2019. Factors identifying aquifers with a high probability of management success. Water International, 44 (3): 354-362.

Laraichi, S.; Hammani, A.; Bouignane, A.

2016. Data integration as the key to building a decision support system for groundwater management: Case of Saiss aquifers, Morocco. Groundwater for Sustainable Development, 2-3: 7-15.

Margat, J.; Gun, J. van der.

2013. Groundwater around the world: a geographic synopsis. CRC. Boca Raton, Fla., US. 341 p.

Mahowald, N.M.

2007. Anthropocene changes in desert area: Sensitivity to climate model predictions. Geophysical Research Letters, 34 (18). DOI: 10.1029/2007GL030472.

Pino, E.; Tacora, P.; Steenken, A.; Alfaro, L.; Valle, A.; Chávarri, E.; Mejía, J.

2017. Efecto de las características ambientales y geológicas sobre la calidad del agua en la cuenca del río Caplina,
Tacna, Perú. Tecnología y Ciencias del Agua, 08 (06): 77-99.

Pino, V.E.; Chávarri, V.E.; Ramos, F.L.

2018. Crisis de gobernanza y gobernabilidad y sus implicancias en el uso inadecuado del agua subterránea, caso acuífero costero de La Yarada, Tacna, Perú. Idesia (Arica), 36 (3): 75-85.

Pino V. E.; Coarita A., F.

2018. Caracterización hidrogeológica para determinar el deterioro de la calidad del agua en el acuifero la yarada media. Revista de Investigaciones Altoandinas -Journal of High Andean Research, 20 (4): 477-490.

Pino, V.E.

2019. El acuífero costero La Yarada, después de 100 años de explotación como sustento de una agricultura en zonas áridas: Una revisión histórica. Idesia (Arica), 37 (3): 39-45.

Pino, E.; Ramos, L.; Avalos, O.; Tacora, P.; Chávarri, E.; Angulo, O.; Mejía, J.

2019. Factors affecting depletion and pollution by marine intrusion in the La Yarada's coastal aquifer, Tacna, Peru. Tecnología y Ciencias del Agua, 10 (5): 177-213.

Pool, M.; Carrera, J.

2010. Dynamics of negative hydraulic barriers to prevent seawater intrusion. Hydrogeology Journal, 18 (1): 95-105.

Post, V.E.A.

2005. Fresh and saline groundwater interaction in coastal aquifers: Is our technology ready for the problems ahead? Hydrogeology Journal, 13 (1): 120-123.

Salgot, M.; Folch, M.

2018. Wastewater treatment and water reuse. Current Opinion in Environmental Science \& Health, 2: 64-74. 
Sanchez, R.; Eckstein, G.

2017. Aquifers Shared Between Mexico and the United States: Management Perspectives and Their Transboundary Nature. Groundwater, 55 (4): 495-505.

Schneider, S.H.; Root, T.L.; Mastrandrea, M.D.

2011. Encyclopedia of Climate and Weather (2. ${ }^{\mathrm{a}}$ ed.). Oxford University Press. 1488 p.

Shiklomanov, A.; Rodda, J.

2004. World Water Resources at the Beginning of the TwentyFirst Century. Cambridge University Press. 452 p.

Singh, S.K.; Zeddies, M.; Shankar, U.; Griffiths, G.A.

2019. Potential groundwater recharge zones within New

Zealand. Geoscience Frontiers, 10 (3), 1065-1072.

Singh, A.

2014. Simulation and optimization modeling for the management of groundwater resources. II: Combined applications. Journal of Irrigation and Drainage Engineering, 140 (4). DOI: 10.1061/(ASCE)IR.1943-4774.0000689.

Thangarajan, M.

2007. Groundwater Models and Their Role in Assessment and Management of Groundwater Resources and Pollution. En M. Thangarajan (Ed.), Groundwater: Resource Evaluation, Augmentation, Contamination, Restoration, Modeling and Management. Springer Netherlands. pp. 189-236.
Vásquez, A.; Vásquez I.; Vásquez, C.

2011. Cosecha de Agua de Lluvia: Y su impacto en el proceso de desertificación y cambio climático. Ed. UNALM. Lima, Perú. 184 p.

Vernoux, J.F.; Horriche, F.; Ghoudi, R.; Abdedaiem, S.; Hamza, M. 2018. Gabes Jeffara Aquifers Management in Relation with Oasien Ecosystems. En: A. Kallel, M.; Ksibi, H.; Ben Dhia,; N. Khélifi (Eds.). Recent Advances in Environmental Science from the Euro-Mediterranean and Surrounding Regions. Springer International Publishing. pp. 599-601.

Wang, Y.J.; Qin, D.H.

2017. Influence of climate change and human activity on water resources in arid region of Northwest China: An overview. Advances in Climate Change Research, 8 (4): 268-278.

WWAP.

2015. Agua para un mundo sostenible: datos y cifras. Informe de las Naciones Unidas sobre los recursos hídricos en el mundo 2015. World Water Assessment Programme. Disponible en: http://www.unesco.org/new/en/natural-sciences/ environment/water/wwap/wwdr/.

Zhang, B.G.

2000. Asymptotic behavior of solutions of certain difference equations. Applied Mathematics Letters, 13 (1): 13-18. 
